ORIGINAL

\title{
EFECTO DEL COBRE SOBRE LA REPRODUCCIÓN EN NOVILLAS LECHERAS DE CUBA
}

\author{
EFFECT OF COPPER ON THE REPRODUCTION \\ OF DAIRY HEIFERS OF CUBA
}

Juan García $D^{* 1}$, Mario C uesta $\mathrm{M}^{1}$, Rodolfo Pedroso $\mathrm{S}^{2}$, Marisol Gutiérrez $\mathrm{P}^{1}$, Angel Mollineda $\mathrm{T}^{1}$, José Figueredo $\mathrm{R}^{3}$

${ }^{1}$ Universidad Central de Las Villas. Facultad de Ciencias Agropecuarias. Carretera a Camajuaní Km 512/2. Santa Clara, Villa Clara. Cuba. ${ }^{2}$ Instituto de Ganadería Tropical (IGAT). Habana, Cuba. ${ }^{3}$ Centro Nacional de Sanidad Agropecuaria (CENSA). San José de las Lajas, La Habana, Cuba. ${ }^{*}$ Correspondencia: juanramon@agronet.uclv.edu.cu

Recibido: Abril 19 de 2006; Aceptado: Noviembre 30 de 2006

\section{RESU MEN}

O bjetivo. Evaluar el efecto del cobre sobre el comportamiento reproductivo de novillas lecheras. Materiales y métodos. El trabajo se realizó en fincas de la provincia de Villa Clara, en dos etapas y con 210 novillas. En la primera se estudiaron 120 novillas divididas en dos grupos de 60 animales cada uno. El grupo A, control y el B, tratado con $2 \mathrm{ml}$ de CuSO $4.5 \%)$ subcutáneamente. En la segunda etapa se estudiaron 90 novillas divididas en tres grupos de 30 animales cada uno, el grupo A, control; el B, fue tratado con $2 \mathrm{ml}$ de $\mathrm{CuSO}_{4}(2.5 \%)$ subcutáneamente y el grupo $\mathrm{C}$, se trató con acupuntura utilizando $0.1 \mathrm{ml}$ de dicho producto subcutáneamente en el punto Pahuai; en ambas etapas los animales del grupo A, no recibieron ningún tratamiento y a los a nimales tratados, grupos B y $C$ se les repitió el tratamiento cada 60 días en dos aplicaciones más. Los análisis hemoquímicos se efectuaron en los laboratorios de la Facultad de Ciencias Agropecuarias de la

Universidad C entral de Las Villas. Resultados. Los animales aumentaron significativamente los niveles del cobre en suero sanguíneo $(p<0.05)$, así como los porcentajes de presentación de celo y de gestaciones con el consiguiente beneficio económico. Conclusión. La aplicación de la cuproterapia tanto alopática como acupunturalmente incrementó la cupremia y la fertilidad de las hembras bovinas tratadas.

Palabras clave: Cobre, hipocupremia, cuproterapia, fertilidad, bovinos, Cuba.

\section{ABSTRACT}

0 bjective. The purpose of this study was to evaluate the effect of copper on the reproductive behavior of dairy heifers. Materials and methods. The work was conducted in farms of Villa Clara province, in two stages and with 210 heifers. In the first one 120 heifers were divided in two groups of 60 animals each one. Group A, control and the group B was treated with $2 \mathrm{ml}$ of CuSO $(2.5 \%)$ subcutaneously. In the second one 90 heifers were studied divided in three groups of 30 animals each one, group A, control; group B was treated subcutaneously with $2 \mathrm{ml}$ of CuSO ${ }_{4}(2.5 \%)$ and the 
group C, was treated with acupuncture using $0.1 \mathrm{ml}$ of this product subcutaneously in the PaHuai point; in both stages the animals included in the group A were not treated and the animals included in group $B$ and $C$ received a repeated treatment every 60 days in two applications more. Results. The animals significantly increased the levels of copper in blood serum $(p<0.05)$, as well as percentages of zeal and gestations presentation with the resulting economic benefit. Conclusion. The application of cuppertheraphy as much allopathical as the acupunctural increase cupperhemic and fertility of the treated bovine females.

Key words: Cupper, hypocupperhemic, cupperteraphy, fertility, bovines, Cuba.

\section{INTRODUCCIÓN}

El cobre desempeña funciones importantes, asociadas directamente con la salud y producción de los rumiantes (1), la deficiencia de cobre en el ganado, ha sido reportada en casi to das las regiones del mundo, siendo uno de los minerales críticos para los rumiantes en pastoreo (2).

La deficiencia de cobre $\left(\mathrm{Cu}^{2+}\right)$ o hipocuprosis es la segunda carencia mineral más frecuente en bovinos en pastoreo en el mundo, después de la de fósforo (3). Pedroso (4) demostró que un $77 \%$ del ganado bovino de Cuba posee deficiencias de $\mathrm{Cu}^{2+}$. Similar situación ha sido reportada por otros autores (5). Existen opiniones que las deficiencias minerales se encuentran asociadas a diversos trastornos de la fertilidad en el ganado bovino criado en pastoreo. En tal sentido algunos autores plantean que el retardo en la aparición de la pubertad, el retardo en la involución del útero, abortos, anestros, retenciones placentarias y repeticiones de servicios en el ganado bovino se deben a la hipocupremia (6 - 8).

Por otra parte, Brem et al. (9) sostienen que en bovinos suplementados con cobre se observaron mayor número de celos, mejores tasas se servicio por concepción e incremento en los porcentajes de preñez. En contraste, otros no obtuvieron respuesta ante el suministro parenteral de cobre con relación al número de servicios por concepción, intervalos parto primer celo y tasas de concepción al primer servicio $(10,11)$.

El objetivo del presente trabajo fue evaluar el efecto la suplementación del cobre por vía parenteral sobre la fertilidad en hembras bovinas en pastoreo con deficiencia de cobre.

\section{MATERIALES Y MÉTODOS}

Sitio de estudio, grupos de pacientes y dosis de cobre. El experimento se desarrolló en dos etapas e incluyeron 210 novillas Siboney, pertenecientes a la granja Santa Clara, provincia de Villa Clara. En la primera se estudiaron las 120 novillas divididas en dos grupos homogéneos de 60 animales cada uno (A y B). El grupo A sirvió de control y el $B$, fue tratado con $2 \mathrm{ml}$ de $\mathrm{CuSO}_{4}(2.5 \%)$ inyectado subcutáneamente, repitiendo dicha inyección cada dos meses hasta completar tres aplicaciones.

En la segunda se estudiaron 90 novillas divididas en tres grupos homogéneos de 30 animales cada uno $(A, B, C)$. El grupo $A$ sirvió de control; el B fue tratado con $2 \mathrm{ml}^{\text {de } \mathrm{CuSO}_{4}}$ $(2.5 \%)$ inyectado subcutáneamente y el grupo C, se trató con acupuntura utilizando $0.1 \mathrm{ml}$ de CuSO ${ }_{4}(2.5 \%)$ inyectable subcutáneamente en el punto Pahuai o Bahuai (espacio lumbosacro). A estos dos últimos grupos se les repitió la inyección cada dos meses hasta completar tres aplicaciones. Para los análisis bioquímicos y hema toló gicos se recolectaron 10 $\mathrm{ml}$ de sangre de la vena coccígea en tubos tipo vacutainer ${ }^{\circledR}$ sin anticoagulante para la obtención del suero.

Análisis hemoquímicos y condición corporal. Los análisis hemoquímicos se realizaron por espectrofotometría de absorción atómica. La evaluación de la condición corporal (CC) se realizó de acuerdo con el método descrito por Parker (12).

Evaluación ginecológica. Se realizó palpación transrectal a to das las hembras para determinar su estado reproductivo y patologías de acuerdo con el método descrito por Holy (13) 
complementando dicho examen con la exploración vaginal mediante espéculo. La evaluación ginecológica se efectuó sistemáticamente con cada aplicación de sulfato de cobre (tres evaluaciones) y la última evaluación se efectuó 30 días después de la última aplicación.

Análisis de los resultados. Los datos se procesaron a través de un paquete estadístico Statgraphics Plus 5.0, aplicándose para las diferencias entre medias de los datos hemoquímicos (ANOVA). Se realizaron comparaciones de proporciones para la presentación de celos y porcentajes de gestación, análisis de varianza y de regresión, así como la aplicación de técnicas no paramétricas.

\section{RESULTADOS}

Las investigaciones hematológicas y hemoquímicas en los animales confirmaron la carencia de cobre en los animales investigados, no se observaron diferencias estadísticas entre los diferentes grupos de tratamientos en los niveles de cobre sérico $(p>0.05)$. En los animales tratados hubo diferencias estadísticas significativas pre y postratamiento en los valores del microelemento $(p<0.05)$.

En la primera etapa se obtuvo un $96.6 \%$ de presentación de celos en grupo que recibió la cuproterapia, en contraste con el grupo control donde solo se alcanzó un 53.3\%. Por otra parte, en el grupo tratado se gestaron 51 animales alcanzándose una concepción de $89.5 \%$ contra las hembras que presentaron celo y del $85 \%$ contra el total de hembras del mismo grupo; resultados superiores a los obtenidos en el grupo utilizado como control donde el porcentaje de gestaciones contra las hembras en celo e inseminadas fue del $68.7 \%$ y $36 \%$ $(p<0.05)$ con respecto al total de hembras de ese mismo grupo (Tabla 1 ).

La condición corporal de las novillas se incrementó en el grupo tratado con la solución de sulfato de cobre; también la cuproterapia empleada incrementó los valores del cobre sérico de manera significativa en grupo que la recibió donde se alcanzó un beneficio económico de US\$ 2.5 por animal tratado, lo que representó una diferencia de US\$1.44 a favor de dicho grupo (US\$ $1=23$ pesos cubanos).

Al comienzo del experimento to das las novillas estaban vacías, pero al segundo tratamiento había más hembras inseminadas (veinticuatro), lo que representó el $43.3 \%$ en el grupo tratado (B); mientras en el grupo testigo (A), en igual período solo se habían servido 6 novillas, representó un $16.6 \%$ de las hembras del grupo $(p<0.05)$.

Al tercer tratamiento, 120 días después de haberse iniciado el estudio se pudo comprobar que en el grupo tratado había 10 animales más inseminados y 34 gestantes, 16 más que en el grupo control, apreciándose una diferencia en ambas variables a favor de los animales tratados. Después, en la cuarta y última observación realizada treinta días posteriores

Tabla 1. Efecto de la administración de una solución de sulfato de cobre $(25 \mathrm{mg} / \mathrm{ml})$ vía parenteral sobre el comportamiento reproductivo en hembras bovinas en pastoreo con celo normal.

\begin{tabular}{lcc}
\hline Indicadores & G rupo control & G rupo tratado \\
\hline Número de animales & 60 & 60 \\
Condición corporal media & 3.6 & 3.9 \\
Valores del cobre sérico (mmol/l) & $11.04 \pm 1.08 \mathrm{a}$ & $13.39 \pm 2.37 \mathrm{~b}$ \\
Hembras que presentaron celo & 32 & 57 \\
Hembras gestantes & 22 & 51 \\
Concepción(\% ) contra las hembras que presentaron celo & $68.7 \mathrm{a}$ & $89.5 \mathrm{~b}$ \\
Concepción(\% ) contra el total de hembras del grupo & $36 \mathrm{a}$ & $85 \mathrm{~b}$ \\
Beneficio económico (US\$ por novillas) & 1.05 & 2.5 \\
\hline
\end{tabular}

$a b=$ medias con diferentes letras en la misma fila difieren significativamente para $p<0.05$. 
al tercer tratamiento al grupo tratado, se diagnosticaron 51 animales gestantes, diferencia apreciable a favor del mismo con respecto al grupo control donde se lograron 22 gestaciones (Figuras 1 y 2 ).

Al analizar los resultados de la segunda etapa (Tabla 2), las proporciones de animales que presentaron estros y los gestantes coincidieron

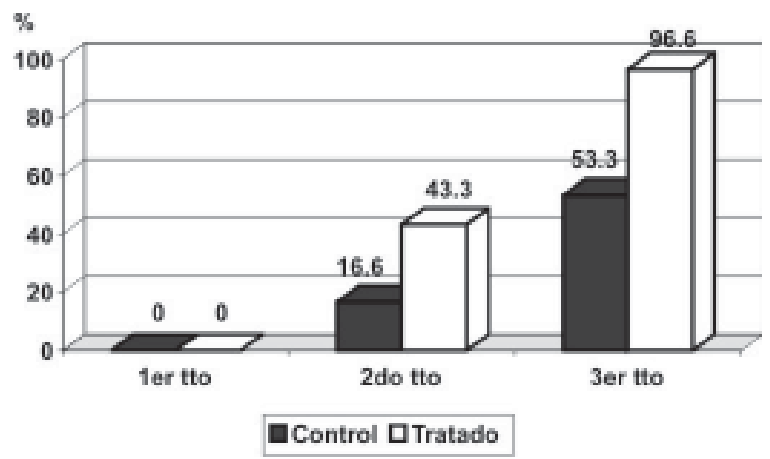

Figura 1. Evolución de las presentaciones de celo e inseminaciones durante el experimento con relación al total de hembras del grupo. con los mejores resultados alcanzados con tratamientos alopáticos y los hormonales más costosos. Al realizar tres aplicaciones con sulfato de cobre tanto por vía subcutánea como en el acupunto Pahuai, se lograron porcentajes superiores de celo y de gestaciones. Además, se redujó el intervalo de anestros y de repeticiones de servicios.

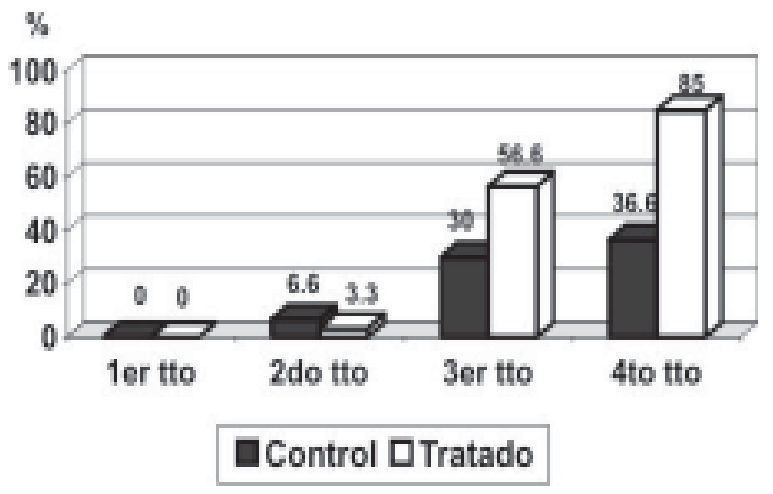

Figura 2. Evolución de las gestaciones con relación al total de hembras del grupo durante el experimento.

Tabla 2. Evolución reproductiva de las novillas tratadas con sulfato de cobre administrado en un punto de acupuntura y alopáticamente.

\begin{tabular}{llllcc}
\hline G rupos & $\mathbf{n}$ & Tratamientos & Cobre (mmol/I) & Celo \% & Gestantes \% \\
\hline $\mathrm{A}$ & 30 & Control & $11.22 \pm 1.08$ & $60 \mathrm{~b}$ & 30 \\
$\mathrm{~B}$ & 30 & $2 \mathrm{mlCuSO}_{4}(2.5 \%)$ & $13.71 \pm 2.14$ & $83 \mathrm{a}$ & 60 \\
$\mathrm{C}$ & 30 & $0.1 \mathrm{mlCuSO}_{4}(2.5 \%)$ BaHui & $13.50 \pm 1.93$ & $80 \mathrm{a}$ & 70 \\
\hline
\end{tabular}

$a b=$ medias con diferentes letras en la misma columna difieren significativamente para $p<0.05$

En la figura 3 se observa el comportamiento del cobre sérico antes y después del tratamiento en los tres grupos estudiados.

Hasta el momento se había palpado un aumento del volumen ovárico en el grupo tratado con la solución de sulfato de cobre en relación con el grupo control lo que debe haber favorecido entre otras cosas el mayor porcentaje de hembras en celo observadas y el mayor porcentaje de concepción alcanzados en el primero.

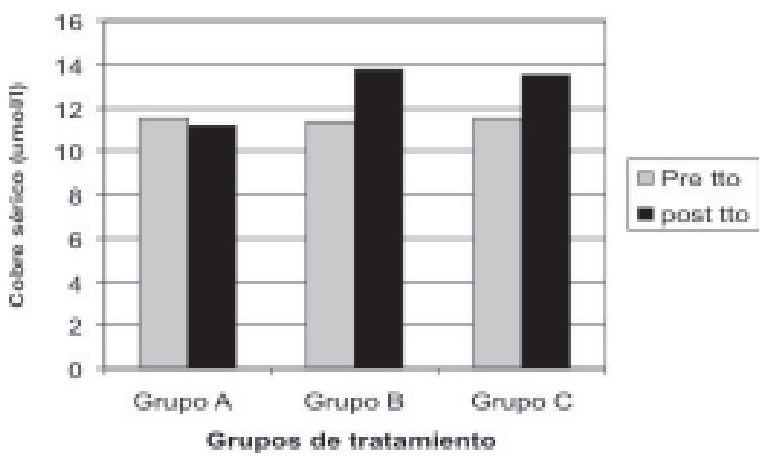

Figura 3. Comportamiento de los valores del cobre sérico, antes y después de la cuproterapia aplicada en los tres grupos de tratamientos. 


\section{DISCUSIÓN}

Los resultados hemoquímicos coinciden con los reportados por otros autores en Cuba (14 17). Estos permanecieron por debajo de 12.6 $\mathrm{mmol} / \mathrm{l}$, valor considerado normal para la especie bovina, coincidiendo con lo planteado por Jagos et al. (18) y Graham (19). Pedroso et al. (8) han reportado el papel de la deficiencia de cobre, su suplementación por vía oral y la respuesta a la misma, lográndose con ella porcentajes superiores de gestaciones con el consiguiente beneficio económico, coincidiendo con los resultados reportados en un estudio de campo realizado con ganado de carne en los Estados Unidos (20). En Cuba la suplementación mineral corregida y en especial la del cobre, por vía oral, en otras áreas de producción ha contribuido a mejorar la fertilidad y productividad del ganado (21 - 24).

Aunque el método tradicional de proveer suplementos minerales, es la combinación de éstos con los concentrados, desafortunadamente los rumiantes en pastoreo reciben pequeñas cantidades de estos. Además, cuando le administran a libre acceso, no se puede controlar el consumo individual, lo que puede ocasionar daños en la salud del animal (25). La suplementación del cobre en forma de mezclas minerales, ha sido recomendada como una forma eficiente de suministrar cobre (26). No obstante, en la prevención y tratamiento de la deficiencia de cobre el método más utilizado es por medio de soluciones inyectables subcutáneas, de manera masiva (27). Estos compuestos inyectables de cobre de liberación lenta constituyen un avance indiscutible en la prevención y tratamiento de la hipocupremia (28).

Estos fármacos suelen ser de elección porque permiten realizar una reserva de $\mathrm{Cu}^{2+}$ en el hígado del animal, no son costosas y al compararlas con la suplementación oral poseen practicidad de manejo y además evitan las interferencias a nivel digestivo (29). La suplementación con cobre por vía parenteral ha sido recomendada como medida profiláctica para prevenir pérdidas económicas (30).

Los incrementos del cobre sérico obtenidos una vez aplicada la cuproterapia están de acuerdo con los obtenidos por diferentes autores (31 34), quienes han demostrado los beneficios de tratar la deficiencia de cobre utilizando compuestos de cobre de liberación lenta por vía parenteral. Igualmente, García et al. (17) al administrar sulfato de cobre por dos vías, subcutánea y en el acupunto Bahuai, lograron en ambos grupos de tratamientos aumentar $(p<0.05)$ los niveles de cobre sérico, el hierro, la hemoglobina, el hematocrito y la condición corporal de los animales tratados.

Según los resultados es posible concluir que los mayores porcentajes de presentaciones de celo y gestaciones observados en los animales tratados obedecen al tratamiento que estos recibieron con el compuesto inyectable de sulfato de cobre. Además, aumentó el índice de condición corporal. García y Cuesta (35) aplicaron por vía parenteral cobre e incrementaron las presentaciones de celos y los porcentajes de gestaciones con un aumento del beneficio económico a favor de los animales tratados.

Los resultados de este trabajo con el tratamiento con $\mathrm{Cu}^{2+}$ inyectable son similares a los de Correa y Lagos (36). Estos últimos estudiaron novillas de primer servicio, con una preñez del $76 \%$ y suplementando cobre por vía inyectable lograron aumentar el índice al $91 \%$, y con el $\mathrm{Cu}^{2+}$ suministrado en bateas (oral) al $82 \%$; 10 que demuestra las ventajas de la administración parenteral de dicho microelemento. Por su parte Ricciardino et al. (37) en novillas Hereford en el primer servicio aumentaron la preñez del $67 \%$ en las testigos, al $83 \%$ en las tratadas con $\mathrm{Cu}^{2+}$ inyectable. Resultados similares habían sido reseñados por Arias (38).

Walter y Marro (39), obtuvieron una notable mejoría en el desempeño reproductivo y por ende en la producción y productividad del ganado bovino lechero, suplementando el cobre por vía parenteral ya que lograron reducir los intervalos parto primer servicio, parto concepción e intervalo parto - parto. Situación similar fue observada por Ruksan (40), Ricciardino et al. (41), Viejo y Casaro (42) y Fader y Marro (43).

La condición corporal (CC) de las hembras bovinas está relacionada con el comportamiento productivo y reproductivo de las mismas (44). En ese sentido algunos autores $(14,15)$ coinciden en informar porcentajes de 
gestación e intervalos entre partos demasiado largos en vacas lecheras con deficiente condición corporal, así como la relación de esta con los indicadores de producción de las mismas. La fertilidad está correlacionada con el peso vivo, los cambios de peso y la CC (46). La relación entre la actividad ovárica y fertilidad de las vacas con su condición corporal ha sido reportada por Pedroso (8). La directa relación entre la condición corporal de las vacas y sus resultados productivos y reproductivos han sido también corroborada por varios autores (47 - 49).

La aplicación de diferentes fármacos en el punto de acupuntura GV-III (Pahuai o Bahuai) ha brindado resultados satisfactorios sobre el comportamiento reproductivo. Los resultados alcanzados en este experimento son similares a los reseñados por G arcía y Cuesta (50). Estos últimos aplicaron sulfato de cobre en el mismo acupunto y aumentaron las presentaciones de celo y las gestaciones. Cuesta et al. (51) lograron mejorar la eficiencia reproductiva y los porcentajes de gestaciones aplicando glucosa hipertónica (50\%) en el acupunto Bahuai. Estos resultados coinciden con los de Kothbauer (52) y Lin et al. (53) quienes inyectaron $2 \mathrm{ml}$ de glucosa hipertónica (20\%) en el acupunto
Pahuai y se obtuvo un aumento en la tasa de ovulación y reducción del intervalo de anestro.

El aumento del volumen ovárico observado en el grupo tratado con la solución de sulfato de cobre con relación al grupo control debe haber favorecido el mayor porcentaje de hembras en celo observadas y de concepción en el mismo. En estudios recientes se ha observado una relación significativa entre el volumen ovárico y la presentación de celo y el porcentaje de concepción (54). En este sentido, observaron correlación significativa $(p<0,01)$ entre la escala de clasificación del VO con la presencia de $C L(r=0,72)$, niveles de $P_{4}(r=0,86)$, frecuencia de atrofia ovárica $(r=0,78)$, exhibición de celo $(r=0,60)$ e incluso con la CC $(r=0,66)$.

En conclusión, los resultados demuestran la necesidad de tratar la deficiencia de cobre en el ganado bovino. En ese sentido, el cobre puede ser suplementado con éxito por la vía parenteral, alcanzando resultados superiores en los indicadores biproducctivos de la especie bovina, lo que se traduce en mejores resultados económicos de las empresas pecuarias.

\section{REFERENCIAS}

1. Huerta BM. Nutrición de rumiantes en pastoreo. Memorias del curso Alternativas de Manejo en Bovinos para Carne en Pastoreo. Chapingo, México. 1997.

2. Huerta BM. Respuesta a la corrección de deficiencias minerales sobre el comportamiento del animal. Memorias II seminario internacional Estrategias de Suplementación a bovinos. Chapingo, México. 1999.

3. Underwood EJ, Suttle NF. The Mineral Nutrition of Livestock. CABI Publishing. London. UK. 1999.

4. Pedroso R. Conferencia Reproducción de la Hembra Bovina. Reunión Nacional de Reproducción, Sancti Spiritus. Cuba. 2005
5. González LE, Lavandeira, Pedroso R. Incidencia de deficiencias minerales en áreas ganaderas de Cuba y utilización de mezclas minerales "abiertas" como méto do de combatirlas. Rev Cub Cienc Vet 1988; 19: 73-80.

6. Farina JN, Farina J L, Farina CM. El desbalance mineral como causa de bajos porcentajes de preñez. Rev Shorthorn 1983; 188: 92-94.

7. Pedroso R, Roller F. Alteraciones metabólicas y carenciales que afectan la reproducción y el desarrollo de la aplicación de las tecnologías de transferencia de embriones y fertilización in vitro. Folleto. CIMA. MINAGRI. C. Habana. 1997. 
8. Pedroso R. Conferencia sobre reproducción de la hembra bovina. XVIII PANVET. Palacio de Las Convenciones La Habana, Cuba. Noviembre de 2002.

9. Brem JJ, Mestre J, Pochon DO, Trulls HE. Alteraciones del ciclo estral provocadas por un alto ingreso de molibdeno en vaquillonas Brangus y respuesta a la suplementación con cobre. FCV UNNE Rev Vet Cuba 2002; 12/13: 1 y 2 .

10. Poole DBR, Walshe MJ. Mechanism of the molybdenum/sulfphur antagonism of copper utilization by ruminants. In: Trace Elements metabolism in man and animals (CF Mills ed.), Edimburgh, Livingstone. 1978; p448.

11. Whitaker DA. A field to asses the effect of copper glycinate injections on fertility in dairy cows Br Vet J 1982; 138: 40-44.

12. Parker R. Body condition scoring of Dairy Casttle. Factsheet AG NES. 1989; 410: 20.

13. Holy L. Biología de la reproducción bovina. Ed. Científico- técnica. La Habana. 1987; $332 p$.

14. García JR. Estudio del estado de salud y producción de una unidad pecuaria con especial referencia al diagnóstico de la condición corporal y su relación con parámetros de producción y salud. Trabajo de diploma. Departamento de medicina veterinaria. Facultad de Ciencias Agropecuarias. Universidad C entral de las Villas, Cuba 2003

15. García JR, Cuesta M. Acupuntura veterinaria en la ganadería agroecológica. II: Acuopuntura en la profilaxis del anestro en novillas. Memorias del II Simposio internacional de ganadería agroecológica Las Tunas, Cuba, 2004.

16. Gutiérrez M. Diagnóstico de los niveles hemoquímicos y hematológicos en vacas lecheras y terneros lactantes. Tesis presentada en opción al título de master en medicina veterinaria preventiva. Departamento de medicina veterinaria. Facultad de Ciencias Agropecuarias. Universidad Central de las Villas, Cuba 2004
17. García JR, Cuesta M, Pedroso R. Administración de sulfato de cobre sobre la hemoquímica, hematología y bioactividad del líquido ruminal en vacas. Rev MVZ Córdoba 2005; 10: 639-647.

18. Jagos P, Bouda J, Kredl F, Pedroso R. Los valores bioquímicos y hematológicos de los animales domésticos y las nuevas expresiones de los resultados. La Habana, Cuba: CIMA; 1982. p.17.

19. Graham TW. Trace element deficiencies in cattle. Vet C lin North Am: Food Anim Pract $1991 ; 7: 153-215$.

20. Clark Y. Nutrición efectiva del cobre para animales de granja. 2000; http:// www. geocites.com/raydelpino

21. Pedroso R, González N, Tarrero R. Reproductive performance and metabolic status of cross breed heifers $(\mathrm{H} \times \mathrm{C})$ fed with sugar cane by - products an confined in corrals. Final Research coordination Meeting. Research Program FAO OIEA. Piracicaba, Brasil, 1991.

22. Ruiz T, G onzález N, Pedroso R. Deficiencias minerales en el ganado bovino en Cuba. IX. Forum de Ciencia y Técnica. CIMA. C. Habana, 1995.

23. Viamonte MI, Rondón G, Fajardo H, Aliaga A, Leyva M, Sánchez M, Constenla L. Procedimientos para el control de la reproducción en rebaños bovinos de carne. Memorias del II Congreso Internacional de Agricultura En Ecosistemas Frágiles y Degradados. Bayamo, Granma, Cuba 2004.

24. Fajardo H, Viamonte MI, Rondón G, Aliaga A, Leyva M, Sánchez M, Constenla L. Procedimientos para el control de la reproducción en rebaños bovinos de leche. Memorias del II Congreso Internacional de Agricultura En Ecosistemas Frágiles y Degradados. Bayamo, Granma, Cuba 2004.

25. Suttle FNJ, Brebner K, McLean, FU Hoeggel. Failure of mineral supplementation to avert apparent so dium 
deficiency in lambs with abomasal parasitism. Anim Sci 1997; 63: 103.

26. Grace $N D$, Lee J. Effect of $\mathrm{Co}, \mathrm{Cu}, \mathrm{Fe}, \mathrm{Mn}$, $\mathrm{Se}$, and $\mathrm{Zn}$ supplementation on elemental content of soft tissues and bone in sheep grazing ryegrass/ white clover pasture. $\mathrm{N} \mathrm{Z}$ J Agric Res 1990; 33: 635-647.

27. Allen WM, Mallinson CB. Parenteral methods of supplementation whit copper and selenium. Vet Rec 1984; 114: 451454.

28. Corbellini CN, BJ Carrillo. Avances en sanidad animal y su aplicación en el diagnóstico y control de enfermedades de bovinos; II Avances en enfermedades tóxicas, nutricionales y metabólicas en bovinos. Rev Arg Prod Anim 1985; 5: 481 504.

29. Costa EF, Giulodori MJ, Titarelli CM, Mattioli GA, Ramírez CE. Intoxicación aguda con cobre en terneros y su posible patogenia. Rev Therios 1999; 28: 167171.

30. Ramírez CE, Tittarelli CM, Mattioli GA, Giuliodori MJ, Puchuri MC. Hipocupremia bovina en 5 partidos de la provincia de Buenos Aires, Argentina. Vet Arg 1997; 14(131): 12-17.

31. Balbuena O, McDowell LR, Stahringer RC. Suplementación con cobre inyectable en terneros y vacas con hipocupremia. Vet Arg 1999; 154: 272-280.

32. Descarga CO, Kloster AM, Davies P, Caffaratti JP. Hipocuprosis y parasitismo gastrointestinal en bovinos de recría en la región subhúmeda de la provincia de Córdoba. Rev Arg Prod Anim 1991; 11: 337-345.

33. Buffarini MA, Homse AC, Cseh S. Efecto de la administración parenteral de cobre y cinc sobre la ganancia de peso y los niveles séricos de cobre y cinc en novillos. Rev Arg Prod Anim 1997; 17, Sup.1: 307.

34. Pechin $G H$, Idiart JL, C seh $S$, Corbellini CN, Moralejo R. Evaluación de dos vías de administración de cobre (oral y parenteral) en bovinos de carne. Vet Arg 1999; 16: 651-657.

35. García JR, Cuesta M. Utilización de un formulado inyectable de Cu SO 4 en al profilaxis del anestro en novillas. III Simposio de Medicina Veterinaria y Zootecnia. Tercera conferencia internacional sobre desarrollo agropecuario y sostenibilidad Santa Clara, Villa Clara, Cuba, 2005.

36. Correa M, Lagos F. Efecto del molibdeno y del cobre en la producción de bovinos para carne en los bajos submeridionales. Rev Arg Prod Anim 1985; 4:199-101.

37. Ricciardino Z, Piccinali RL. Efectos de la suplementación parenteral con minerales $(\mathrm{C} u, \mathrm{Se}, \mathrm{P})$ y vitaminas (E y D), sobre parámetros productivos en Vaquillas sobre pasturas naturales. Rev Arg Prod Anim 1998; Sup 1: 335.

38. Arias $A A$, Peruchena $C D$, Manunta $O A$, Slobodzian A. Experiencias de suplementación mineral realizadas en la E.E.A. Corrientes (El Sombrerito). Rev Arg Prod Anim 1985; 4: 57-70.

39. Walter O, Marro O. Efecto de la administración de cobre y selenio inyectable sobre el comportamiento reproductivo de la vacas lecheras www.inta.gov.ar/manfredi/info, 2003.

40. Ruksan BE, Díaz JC, Lagos F. Efecto de la suplementación de cobre y selenio sobre la cupremia, glutation peroxidasa y fertilidad en vaquillonas. Rev Arg Prod Anim 1988; 8 Supl 1: 156.

41. Ricciardino $M$, Medus $P, G$ ómez $M$, Ruksan B. Producción Animal. Informe Técnico No. 3 EEA. INTA Concepción del U ruguay. 1991.

42. Viejo RE, Casaro AP. Efectos de la suplementación con cobre sobre la ganancia de peso, cobre hepático y plasmático en terneros. Rev Arg Prod Anim 1993; 13: 97-105. 
43. Fader $\mathrm{O} \mathrm{W}, \mathrm{M}$ arro $\mathrm{O}$. Efecto del tratamiento con cobre y selenio inyectable sobre el comportamiento reproductivo de animales lecheros en la región central de Córdoba. Ciencia Veterinaria. 1998; 28: 3-9.

44. Moncada E. El clima, la nutrición y la reproducción de bovinos en regiones tropicales. En: Memorias del II Seminario Internacional «Manejo de la reproducción en condiciones tropicales». CIPEC. Cartagena de Indias, Colombia 1994.

45. Cuesta M, Cuesta A, G il L, García JR. Condición corporal de las vacas lecheras y su relación con indicadores productivos, de reproducción, de la calidad de la leche y parámetros de bioactividad ruminal y sanguíneos. Segunda conferencia internacional sobre desarrollo agropecuario y sostenibilidad Santa Clara, Villa Clara, Cuba, 2003.

46. Carulla J. El rumen y la salud. En: Memorias del VII Simposio Nacional y tercero internacional de Medicina Veterinaria y Zootecnia. Universidad de Córdoba. Montería, Colombia 1998.

47. López R. Condición corporal: un criterio importante en el manejo de las vacas lecheras. X Jornada Científica por el 20 Aniversario del CIMA. Resúmenes p89 Revista ACPA. 1995; 1 y 2: 31.

48. Álvarez JL. La condición corporal en la hembra bovina. Rev Sal Anim 1997; 19: 37-46.
49. Bono G. Lactación y el reinicio de la actividad reproductiva durante el postparto en vacas lecheras. Agro - Ciencias. 1997; 13: 19-29.

50. García JR, Cuesta M. Diagnóstico y tratamiento de la deficiencia de cobre y su efecto sobre la reproducción y el estado de salud de las hembras bovinas. Memorias del II Congreso Internacional de Agricultura En Ecosistemas Frágiles y Degradados. Bayamo, Granma, Cuba, 2004.

51. Cuesta M, García JR, Lazo M, Gutierrez M, Martínez E, Figueredo M. Acupuntura en la profilaxis del anestro posparto en novillas y cerdas mediante la utilización de una formulación inyectable de $\mathrm{CuSO}_{4}$ en el acupunto Bahuai. Tercera conferencia internacional sobre desarrollo agropecuario y sostenibilidad Santa Clara, Villa Clara, Cuba, 2005.

52. Kothbauer OA. Texbook on Veterinary AP, Welsermuhi Wels Austria 1995; p334.

53. Lin JH Rogers, PAM, Yamada H. The scientific basis of $C$ hinese Herbal Medicine. China Veterinary Science. Inc. California. 1997.

54. G onzález-Stagnaro N, Madrid-Bury, Rojas N. Clasificación del volumen ovárico de vacas lecheras en anestro posparto. http:/ / www.geocites.com. 1999. 